DR LIANGJUN YIN (Orcid ID : 0000-0003-1772-2213)

PROFESSOR XIN XU (Orcid ID : 0000-0001-6547-255X)

Article type : Article

\title{
Direct observation of Eu atoms in AIN lattice and the first-principles simulations
}

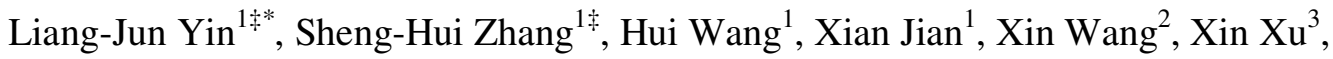

Ming-zhen Liu ${ }^{4}$, Chang-Ming Fang ${ }^{5 *}$

${ }^{1}$ School of Materials and Energy, University of Electronic Science and Technology of China, 2006 Xiyuan Road, Chengdu, 611731, P.R. China

${ }^{2}$ National Engineering Research Center of Electromagnetic Radiation Control Materials, University of Electronic Science and Technology of China, 2006 Xiyuan Road, Chengdu, P.R.

China

${ }^{3}$ Laboratory of materials for energy conversion, Department of Materials Science and

Engineering, University of Science and Technology of China, Hefei, P.R. China

${ }^{4}$ Center for Applied Chemistry, University of Electronic Science and Technology of China,

2006 Xiyuan Road, Chengdu, 611731, P.R. China

${ }^{5}$ Brunel centre for Advanced Solidification Technology (BCAST), IMM, Brunel University

London, Uxbridge, Middlesex, UB8 3PH, United Kingdom

*Corresponding Author: ylj@mail.ustc.edu.cn (Liang-Jun Yin);

This article has been accepted for publication and undergone full peer review but has not been through the copyediting, typesetting, pagination and proofreading process, which may lead to differences between this version and the Version of Record. Please cite this article as doi: $10.1111 /$ jace. 15912

This article is protected by copyright. All rights reserved. 
Changming.Fang@ brunel.ac.uk (Chang-Ming Fang).

\begin{abstract}
Rare-earth metal $(\mathrm{Eu})$ doped aluminum nitride has potential application as luminescence materials due to its unusual mechanical and physical properties, as well as high chemical stability. Here we investigate the energetics, local structure and optical and electronic properties by means of a combination of experimental observations (XDR, SEM, HR-TEM and XANES) and first-principles simulations. Present study has revealed that Eu ions are likely to be co-doped with $\mathrm{O}$ in the form of Eu-O pairs. Eu ions or Eu-O pairs favor participation at the surfaces of the AlN crystallites. Our analyses show dependences of the Eu valence and electronic/optical properties on the local chemical composition and structure. The obtained information helps us to realize tuning of the optical properties of the luminescent materials via composition and site occupation modification.
\end{abstract}

Keywords: AlN, Eu site, phosphor, first-principle calculation.

\title{
I. Introduction
}

Light-emitting-diodes (LEDs) are replacing conventional lighting sources due to their multiple advantages, such as high light conversion ratios, long lifetime, low energy consumption, high reliability, and environmental-friendliness ${ }^{1,2}$. (Oxy)nitrides phosphors have a strong absorption in the UV-blue band, adjustable emission color and good thermal quenching and therefore, at present are developed increasingly ${ }^{3,4}$. Among them, aluminum nitride (AlN) has been of particular interest due to its high electrical insulation (energy gap of This article is protected by copyright. All rights reserved. 
$6.2 \mathrm{eV}$ ), high thermal conductivity, low thermal expansion coefficients as well as high chemical stability ${ }^{5,6}$, and is considered as a potential host for photoluminescence and electroluminescence devices and for LED and FED devices ${ }^{7}$.

There have been experimental efforts to understand the luminescent properties of AlN based phosphors ${ }^{8-14}$. It was demonstrated that Eu, Si co-doped AlN phosphor shows a strong blue luminescence by UV or electron excitation ${ }^{13,15}$. This co-doping which remains the chemical valence balance, promotes Eu incorporation into the AlN lattice and the obtained phosphor remains the wurtzite structure. In our previous study, we prepared Eu solely doped AlN samples by different synthesis methods (solid-state reaction (SSR), carbothermal reduction $(\mathrm{CR})$ and gas-reduction nitridation $(\mathrm{GRN}))$. The experimental observations showed that the luminescence properties are highly dependent on the oxygen content in the final samples ${ }^{16-18}$. Structural analysis showed that the AlN: Eu phosphor with a low oxygen content is dominated by the AlN wurtzite structure via the CR and GRN methods. In general, the large radius mismatches between the $\mathrm{Al}^{3+}(0.39 \AA, 4 \mathrm{CN})$ and $\mathrm{Eu}^{2+}(1.17 \AA, 6 \mathrm{CN})$ or $\mathrm{Eu}^{3+}$ $(0.95 \AA, 6 \mathrm{CN})$ ions ${ }^{19}$ indicate high energy costs for the replacements of $\mathrm{Al}^{3+}$ by $\mathrm{Eu}$ ions in the doped samples. Up to now, knowledge about these materials, e.g. the site occupations of Eu ions in the wurtzite AlN lattice is still limited. In this aspect, theoretical methods, especially parameters-free first-principles approaches are helpful. First-principles calculations have been applied successfully to analyze the electronic structure of aluminum nitride ${ }^{20}$ and to investigate the structural models of aluminum oxynitride ${ }^{21}$. Pentaleri and co-workers studied substitutional impurities in zinc-blende aluminum nitride ${ }^{22}$. Recently ab initio density functional theory was applied successfully in study of the doping of Eu in scintillation and This article is protected by copyright. All rights reserved. 
luminescent materials ${ }^{23-25}$. In the current paper, we prepared the high-pure AlN: Eu phosphors by gas-reduction-nitridation (GRN) method and investigated the preferred sites of Eu ions in the AlN samples by a combination of multiple experimental techniques (SEM, EXAFS/XANES, STEM) and first-principles calculations. The ab initio simulations were performed for Eu doped in (wurtzite) AlN bulk and at its (110) and (0001) surfaces. The influence of oxygen on the Eu doping was addressed, considering the fact that oxygen is always unavoidable in the sample preparations. The obtained information here is helpful/useful to understand the mechanism of the luminescence properties, to obtain inspiration for AlN based phosphors doped with other large (rare-earth) ions and further to search new luminescence materials of desirable properties.

\section{Experimental Sections and First-Principle Simulations}

2.1. Synthesis. Phosphors with a chemical composition of $\mathrm{AlN}$ : $0.5 \mathrm{~mol} \%$ Eu were prepared by the gas-reduction-nitridation method. The powder mixture of $\gamma-\mathrm{Al}_{2} \mathrm{O}_{3}$ and $\mathrm{Eu}_{2} \mathrm{O}_{3}$ were fired in a horizontal alumina tube furnace at $1400{ }^{\circ} \mathrm{C}$ for $3 \mathrm{~h}$ under a flowing gas mixture of 1 1/min $\mathrm{NH}_{3}$ and $15 \mathrm{ml} / \mathrm{min} \mathrm{CH}_{4}$. The as-prepared products were post-annealed at $1900{ }^{\circ} \mathrm{C}$ for 2 $\mathrm{h}$ in a carbon furnace under flowing nitrogen gas, to further improve its crystallinity.

2.2 Characterization. The phase was analyzed by an X-ray diffractometer (Model PW1700, Philips Research Laboratories, Eindhoven, the Netherlands) using $\mathrm{Cu} \mathrm{K}_{\alpha}$ radiation. Powder morphologies were observed by scanning electron microscopy (SEM) (Model JSM-6390LA, JEOL, Tokyo, Japan). The nitrogen/oxygen content was determined using a nitrogen/oxygen Analyzer (ModelTC-436, LECO, Tokyo, Japan. Elemental analysis was carried out using

This article is protected by copyright. All rights reserved. 
Induced Couple Plasma Optical Emission Spectroscopy (ICP-OES, PerkinElmer Optima 5300, USA). Cathodoluminescence spectra (CL) were recorded in the Scanning Electron Microscope (SEM) equipped with a CL accessory (Sirion200, FEI, USA). The X-ray absorption near edge structure (XANES) of $\mathrm{Eu} \mathrm{L}_{3}$-edge was measured at the beamline of BL14W1 at Shanghai Synchrotron Radiation Facility with an electron beam energy of 3.5 GeV. Z-contrast Scanning transmission electron microscopy (STEM) was performed using high angle annular-dark field scanning electron microscopy (HAADF-STEM, JEM-ARM 200F). HAADF simulations were performed using Dr. Probe software.

2.3 Super cell method for first-principle simulations: To describe the effect of dilute $\mathrm{Eu}$ atoms/ions and Eu-O pairs in bulk AlN, we employed a supercell with dimensions of $4 a_{0} \times 4 a_{0} \times 2 c_{0}\left(a_{0}, c_{0}\right.$ are the lattice parameters of a pristine unit cell of hexagonal AlN). This hexagonal supercell has axis lengths of about $a=12.51$ and $c=10.03 \AA$ and contains 128 atoms (64 $\mathrm{Al}$ and $64 \mathrm{~N}$ ). For the AlN (110) surface, we build a supercell which has in plane axis lengths of $a=4 a_{0}$ and $b=2 c_{0}$. This cell has 12 atomic layers (AL) and contains in total 192 atoms (96 Al and $96 \mathrm{~N})$. A thick slab of vacuum (>17 ̊̊) was used to avoid interface interactions. A single Eu atom replaced an $\mathrm{Al} \mathrm{a)} \mathrm{in} \mathrm{the} \mathrm{center,} \mathrm{b)} \mathrm{at} \mathrm{the} \mathrm{surface} \mathrm{and} \mathrm{c)} \mathrm{second} \mathrm{layer} \mathrm{of} \mathrm{the}$ surface (subsurface). Similarly, Eu and O co-doping replaced an Al-N pair a) at the center and at the surface of the slab. Test calculations for the polar AIN (0001) surfaces were performed for a supercell of a hexagonal lattice with $a=b=2 a_{0}$ in plane. There are $16 \mathrm{Al}$ of atoms or 64 atoms $(32 \mathrm{Al}$ and $32 \mathrm{~N})$ in total. The vacuum has a length of about $20 \AA$. One Eu atom replaces one $\mathrm{Al}$ in the center and at the two terminal surfaces to get some conceptual results about the preference of Eu occupation.

This article is protected by copyright. All rights reserved. 
2.4 Computational technique and settings: We employed the first-principles code VASP (Vienna $A b$ initio Simulation Program) ${ }^{26,27}$, which is based on the density functional theory (DFT) within the Projector Augmented-Wave (PAW) method ${ }^{28,29}$. The Generalized Gradient Approximation (GGA) was used for the exchange and correlation energy terms. ${ }^{30}$ The cut-off energy of the wave functions was $550.0 \mathrm{eV}$. The cut-off energy of the augmentation wave functions was $700.0 \mathrm{eV}$. Such high cut-off energies provided reliability to describe the rather localized $\mathrm{O} / \mathrm{N} 2 \mathrm{~s}, 2 \mathrm{p}$ and Eu $4 \mathrm{f}$ orbitals. The energy differences were converged within $10^{-5} \mathrm{eV}$. The forces were converged within $10^{-3} \mathrm{eV} / \AA$. The electronic wave functions were sampled on dense grids e.g. a $4 \times 6 \times 1$ grid with 6 to 12 irreducible $k$-points depending on the symmetry, for the super cells of AlN(110) surface systems with Eu or Eu-O doping in the Brillouin zone (BZ) of the crystals using the Monkhorst and Pack method ${ }^{31}$. The localized Eu 4f states were described using the Hubbard $\mathrm{U}(U=2.5 \mathrm{eV})$ approach ${ }^{24,25}$. Tests of $k$-mesh density and cut-off energies showed a good convergence ( $\sim \mathrm{meV} /$ atom $)$.

\section{Results and Discussions}

\subsection{Experimental observations}

3.1A. Phase, composition and morphology. As seen in Fig. S1 of the supporting information, our Rietveld refinement parameters $\left(\mathrm{R}_{\mathrm{P}}=7.37 \%, \mathrm{R}_{\mathrm{WP}}=9.93 \%\right)$ confirm the phase purity of AlN: Eu powders. By using the induction coupled plasma method and the oxygen-nitrogen analysis, the actual chemical composition of AlN: Eu phosphor is determined as $\mathrm{Eu}_{0.0033} \mathrm{Al}_{0.9962} \mathrm{O}_{0.0156} \mathrm{~N}_{0.9840}$. The particle size of the $\mathrm{AlN}$ : Eu sample is reflected by the SEM images seen in Fig. S2 of the supporting information. The size distribution is non-uniform and covers the range of $1-10 \mathrm{um}$.

This article is protected by copyright. All rights reserved. 
3.1B. Optical properties. Instead of photoluminescence, cathodoluminescence measurement owns a powerful excitation source and can motivate the phosphor to exhibit plentiful emission. Fig. 1 shows the CL spectra of the AlN: Eu powder. As seen, AlN: Eu phosphors show broad green emission band at about $530 \mathrm{~nm}$ under electron irradiation, which is undoubtedly ascribed to the $4 \mathrm{f}^{6} 5 \mathrm{~d} \rightarrow 4 \mathrm{f}^{7}$ transition of $\mathrm{Eu}^{2+}$. The full width at half maximum (FWHM) of the band is quite broad ( 92 nm), which greatly exceeds the value in AlN: Eu, Si $(55 \mathrm{~nm})^{13}$. As shown in Fig. 1, the brightness of AlN: Eu increase as the anode voltage increases from of $5 \mathrm{kV}$ to $20 \mathrm{KV}$. This enhanced intensity as a function of anode voltage is ascribed to the increased number of excited $\mathrm{Eu}^{2+}$ ions resulting from an increase in the penetration depths of the electron beam as the applied voltage is increased.

In Fig. 1, there are two red emissions around 630 and $660 \mathrm{~nm}$ in the CL spectrum. These emissions can be assigned to $\mathrm{Eu}^{3+}$ and $\mathrm{Eu}^{2+}$ intra-4f transitions. Unlike the absence of $\mathrm{Eu}^{3+}$ in AlN with Eu, Si co-doping samples ${ }^{32}$, our results indicate that there are $\mathrm{Eu}^{3+}$ ions companying $\mathrm{Eu}^{2+}$ in the crystal lattice. The coexistence of $\mathrm{Eu}^{2+}$ and $\mathrm{Eu}^{3+}$ is clearly identified by the XANES analysis as shown in Fig. 2, where two peaks can be clearly seen at about 6977 and $6984 \mathrm{eV}$ due to the divalent and trivalent oxidation states of Eu, respectively ${ }^{33}$ as well as the coexistence of $\mathrm{Eu}^{2+}$ and $\mathrm{Eu}^{3+}$ in the $\mathrm{Eu}$ doped polycrystalline $c$ - $\mathrm{BN}$ specimens ${ }^{34}$. The simultaneous presence of $\mathrm{Eu}^{2+}$ and $\mathrm{Eu}^{3+}$ results in the overlapping green/red emission spectra.

This article is protected by copyright. All rights reserved. 


\subsection{Direct observation of Eu sites.}

For a low doping crystal $(0.5 \% \mathrm{Eu})$, the exact doping structure could only be resolved via direct viewing in the atomic scale. By taking advantage of the atom-resolved Cs-corrected STEM, we are able to observe individual heavy atoms, such as the rare-earth atoms in the crystal lattice which can be highlighted in the Z-contrast HAADF (High-angle annular dark-field) images. Fig. 3 (a) shows a typical atomic-resolution STEM image of AIN: Eu. The scattering strength of nitrogen is relatively weak but along the (010) plane, the atomic dumbbell consisting of $\mathrm{Al}$ and $\mathrm{N}$ atoms is still clearly recognized. Thus, we can directly determine the atomic site of the Eu dopants from the images. The atomic columns containing Eu dopants are observed as the brighter spots, and one can see that the Eu dopants are dispersed along the boundary. In the high-magnification atomic resolution STEM image of Fig. 3 (b), the single Eu dopant evidently substitutes the Al site in edge of the AlN crystallites. As shown in Fig. 3 (c), the arrangement of atoms in AlN: Eu matches with the intensity file from the box in Fig. 3 (b). This bright feature at the $\mathrm{Al}$ site is quantitatively reproduced in the simulated image (Fig. 2 (d)), where a single Eu atom has been substituted for an $\mathrm{Al}$ atomic site. In addition to the Eu sites along the boundary, Eu ions can also replace $\mathrm{Al}$ ions close to the boundary, as seen in Fig. 3 (e, f). This may origin from the easily released crystal strain near the boundary.

Overall, our experiments have shown that there are simultaneous presence of $\mathrm{Eu}^{2+}$ and $\mathrm{Eu}^{3+}$ in the AlN: Eu sample. To get insight into the phenomenon, we performed first-principles simulations.

This article is protected by copyright. All rights reserved. 


\subsection{Theoretical simulations}

3.2A. Bulk AIN, EuO and EuN. Our calculation results for the bulk properties of the related compounds, AlN, EuO and EuN are shown in Table I.

(Some references are present in Table $1^{23,24,25,35,36}$ ).

As shown in Table I, the obtained lattice parameters for the bulk AlN, EuO and EuN are close to the experimental values within $1 \%$ error. The present calculations show that wurtzite AlN is more stable than the cubic phase with an energy difference of about $0.45 \mathrm{eV} / \mathrm{AlN}$. The calculations also show that $\mathrm{EuO}$ with table salt structure has a local magnetic moment of $s=7$, whereas the spin state is $s=6$ for the EuN. In order to have a good understanding about the spin states of $\mathrm{Eu}$ in $\mathrm{EuO}$ and $\mathrm{EuN}$, we performed spin-polarization calculations using the fixed spin approach for both $\mathrm{EuO}$ and EuN with table salt structure. The calculated relationship between the cohesive energy and local magnetic moment is shown in Fig. 4. Clearly, the most stable spin state is $s=7 / 2$ for $\mathrm{EuO}$, whereas, it is $s=6 / 2$ for EuN. These results agree with the ionic model: $\mathrm{Eu}^{2+}$ with $4 \mathrm{f}^{7}$ configuration in $\mathrm{EuO}$ and $\mathrm{Eu}^{3+}$ with $4 \mathrm{f}^{6}$ configuration in EuN.

3.2B. Dilute Eu doping and Eu-O co-doping in bulk AIN. We first address the calculations, where a single Eu atom doping and an Eu-O pair co-doping in AlN bulk are discussed, respectively. The calculated results are shown in Table II. Fig. 5 shows the calculated total density of states (tDOS) for the related systems, and the partial density of the related Eu $4 \mathrm{f}$ states. The tDOS of wurtzite AlN is included for comparison.

As shown in Table II, the calculations show that replacing one $\mathrm{Al}$ by Eu costs about $2.6 \mathrm{eV}$ with respect to the parent binaries $\mathrm{AlN}$ and EuN. A Eu-O co-doping costs about $4.5 \mathrm{eV}$ when $\mathrm{Eu}$ and $\mathrm{O}$ are set far-away and about $3.6 \mathrm{eV}$ when Eu and $\mathrm{O}$ is nearby in AlN.

This article is protected by copyright. All rights reserved. 
As shown in Fig. 5 (top row), AlN is calculated to be a wide gap insulator with the calculated energy gap being about $4.2 \mathrm{eV}$, which is smaller than the experimental value. This is not unusual for the density functional theory. ${ }^{37}$ The $\mathrm{N} 2$ s states form a broad band in the energy range between $-13.1 \mathrm{eV}$ to $-10.5 \mathrm{eV}$ (Fermi level is set to be zero $\mathrm{eV}$ ). The valence band which is dominated by $\mathrm{N} 2 \mathrm{p}$ states is in the energy range between $-5.1 \mathrm{eV}$ to zero $\mathrm{eV}$, whereas the conduction band is about $4.2 \mathrm{eV}$ above the valence band. The present results agree with the former theoretical calculations ${ }^{21,22}$.

The tDOS curves of the Eu and Eu-O doped systems are very similar to that of AlN bulk. For EuO co-doped system (Eu-O is close), there is a sharp peak at about $2.1 \mathrm{eV}$ above the valence band. This peak is occupied by electrons. Eigencharacter analysis shows that this peak belongs to the $\mathrm{Eu} 4 \mathrm{f}^{7}$ states for the majority electrons. Meanwhile, the unoccupied $\mathrm{Eu} 4 \mathrm{f}$ states are at upper energy (about $10 \mathrm{eV}$ ) above the Fermi level (not shown). This result corresponds well to the $\mathrm{Eu}^{2+}$ configuration (Table II). The partial density of the Eu 4f states for the Eu doping system (Fig. 5) is more complicated and showed disperse nature. The pDOS of the Eu 4f states for the majority electrons (black lines in third row) consists mainly of two parts. The lower part is in the valence band, ranging from about $-2.5 \mathrm{eV}$ to $-0.5 \mathrm{eV}$, whereas the upper part is unoccupied and positioned at $0.4 \mathrm{eV}$ above the valence band. There are also some small Eu $4 \mathrm{f}$ contributions just below the Fermi level. These disperse nature of the Eu $4 \mathrm{f}$ states indicates strongly interaction between $\mathrm{Eu} 4 \mathrm{f}$ and $\mathrm{N} 2 \mathrm{p}$ states, in contrast to the case with Eu-O co-doping where the Eu 4f states are strongly localized.

This article is protected by copyright. All rights reserved. 
3.2C. Eu at the AIN surfaces. As mentioned before, the ground state of AlN has the wurtzite structure (Fig. S3a) with a hexagonal lattice with $a_{0}=3.11 \AA, c_{0}=4.98 \AA^{38}$. In this structure each $\mathrm{Al}$ is in a tetragonal coordination by four $\mathrm{N}$. It is possible to obtain non-polar surfaces by cleaving the crystal along (110) orientation (Fig. S3b) ${ }^{38}$. A smooth cleavage of AlN along its (0001) orientation produces one Al-terminated and one N-terminated surface. Therefore, a smooth AlN (0001) surface is polar. Both experimental and theoretical studies showed reconstruction of the $\mathrm{Al}$ (0001) surfaces ${ }^{38-41}$. Our goal here is for understanding the Eu preference in the bulk or at the surfaces of AlN. We first chose the non-polar AlN (110) surface as the example. Furthermore, we also investigate the Eu preferred sites using a slab containing smooth AlN (0001) surfaces considering the fact that the surfaces are composed of Al-N or N-Al double atomic layer and, therefore, are weak/moderate in polarity.

Figure 6 shows the single Eu replacements of $\mathrm{Al}$ and $\mathrm{Eu}: \mathrm{O}$ co-doping at different site. The calculated energetics for one Eu atom/ion and one Eu-O pair at different positions at/in AlN (0001) surfaces and (110) are shown in Table III.

First we discuss the calculations for the non-polar AlN (110) surface. As shown in Table III, the total energy calculations show that Eu prefers the surfaces site. The energy difference is about $3.05 \mathrm{eV}$ for one Eu doping at the AlN surface, which is notably larger than that of one $\mathrm{Eu}$ doping at the subsurface. Moreover, Eu-O co-doping at the AlN (110) surface is much favored with an energy difference as $3.2 \mathrm{eV}$. This type of Eu preferential behavior at the surface is understandable considering the large Eu ionic size as compared with an $\mathrm{Al}$ ion. Meanwhile, the $\mathrm{Eu}$ in the center of the slab is connected to four $\mathrm{N}$ with Eu-N bond-length $2.24(\times 2), 2.27$ and $2.30 \AA$ A which are just slightly different from those (Eu-N: $2.22(\times 3), 2.26 \AA)$ for the Eu in AlN This article is protected by copyright. All rights reserved. 
bulk, which comes from the broken symmetry and the surface influence. The calculations show that the local magnetic moments or spin states of the Eu in the center, at the second layer and at the surface of the AlN slab. The Eu at the center of the AlN slab has its spin state $s=6.26 / 2$, which is slightly larger than that in Eu in AlN bulk (6.04/2).

Structural optimization and electronic structure calculations show that for a Eu-O pair in the center or at the surface of AlN, the spin state for $\mathrm{Eu}$ is $s=7 / 2$, corresponding to the $\mathrm{Eu}^{2+}$ model.

Fig. 7 shows the calculated total density of states for Eu (first row) and Eu-O (third row) at AlN surface. The related partial densities of the Eu $4 \mathrm{f}$ states are also included. Clearly, in the Eu-O pair co-doped case, the Eu $4 \mathrm{f}$ states are fully spin-polarized. The occupied $\mathrm{Eu} 4 \mathrm{f}^{7}$ states for majority electrons form a sharp peak at about $2 \mathrm{eV}$ above the valence band, whereas the unoccupied $\mathrm{Eu} 4 \mathrm{f}$ states also form a sharp peak at about $4.5 \mathrm{eV}$ above the occupied $4 \mathrm{f}$ states. There are surface states in the range between about $2.3 \mathrm{eV}$ to above the valence band to the bottom of the conduction band of the bulk AlN.

\subsection{Discussions and summary}

Our study by means of a combination of multiple experimental observation and first-principles simulation has shown that Eu ions or Eu-O pairs favor at the surface of the grain boundary of the AlN particles. O bonded to Eu is a key point to determine the valence and electronic/optical properties of Eu. Single Eu substitution is 3+ valent while Eu ions exhibit a $2+$ valence for Eu-O pairs which was confirmed by the analysis of the oxygen content that $0.60 \mathrm{wt} \%$ of oxygen amount is present in AlN: Eu phosphor.

This article is protected by copyright. All rights reserved. 
Overall, single Eu doping exhibits a 3+ valence, which is responsible for the sharp red emission in AlN: Eu phosphor. Eu ions are likely to get bounded to form Eu-O pair. With the help of Eu-O codoping, $\mathrm{Eu}^{2+}$ is stable as it has a full filled $4 \mathrm{f}$ orbitals $\left(4 \mathrm{f}^{7}\right)$, which results in the broad green emission band of $4 \mathrm{f}-5 \mathrm{~d}$ electron transitions. Based on the crystal field theory, the split of $5 \mathrm{~d}$ orbitals of $\mathrm{Eu}^{2+}$ is affected by the type of ligands and the arrangement of the ligands around the $\mathrm{Eu}^{2+}$. Owing to the higher formal charge of $\mathrm{N}^{3-}$ compared with $\mathrm{O}^{2-}$ and the lower electronegativity of nitrogen (3.04) compared with oxygen (3.44), some $\mathrm{Eu}^{2+}$ doped pure nitride phosphors show emission peaks situated in the red region, such as $610 \mathrm{~nm}$ for Eu doped $\mathrm{SrAlSiN}_{3}(\mathrm{Sr}-\mathrm{N}: \sim 2.7 \AA$, C.N. $=10)$ and $620 \mathrm{~nm}$ for Eu doped $\mathrm{Sr}_{2} \mathrm{Si}_{5} \mathrm{~N}_{8}(\mathrm{Sr}-\mathrm{N}: \sim 2.8 \AA, \mathrm{C} . \mathrm{N}$. $=8-9)^{42,43}$. Regarding to the preferable Eu-O co-doping at the boundary of AlN: Eu phosphor, low coordination number, asymmetry coordination and $\mathrm{O}$ defects would possibly result in a weaker crystal-field splitting and consequently a green emission. Note that it cannot be concluded that Eu stay in the center or subsurface of AlN crystal due to possible local defects around $\mathrm{Eu}^{2+}$, as seen in Fig. 3 (e). Here we briefly summarize our investigations into a schematic diagram of Fig. 8.

\section{Conclusions}

Our findings indicate that even dopant atoms with extremely large size mismatch can be incorporated into host crystal lattice through Eu substitution $\mathrm{Al}$ at or near the surface. Single Eu prefers to remain $3+$ valent due to its large ionic size while Eu owns a 2+ valence when Eu-O co-doping is achieved as $\mathrm{Eu}^{2+}$ has fully filled $4 \mathrm{f}$ orbitals $\left(4 \mathrm{f}^{7}\right)$. Expectedly, we may be 
able to control unique functionalities in largely mismatched systems by tuning the $\mathrm{Eu}^{2+} / \mathrm{Eu}^{3+}$ ratios in the lattice via manipulating samples' particle size distribution, chemical composition and site occupation modification.

\section{Acknowledgements}

This research was supported by the National Natural Science Foundation of China (Grant No. 51302029), the Fundamental Research Funds for the Central Universities (Grant No. ZYGX2015J110).

\section{References}

1. Taguchi T. Present status of energy saving technologies and future prospect in white LED lighting. Ieej T Electr Electr. 2008; 3:21-26.

2. Berencen Y, Carreras J, Jambois O, Ramirez J M, Rodriguez J A, Dominguez C, et al. Metal-nitride-oxide-semiconductor light-emitting devices for general lighting. Opt Exp. 2011; 19:A234-A244.

3. Lee S, Sohn K-S. Effect of inhomogeneous broadening on time-resolved photoluminescence in $\mathrm{CaAlSiN}_{3}: \mathrm{Eu}^{2+}$. Opt Lett. 2010; 35:1004-1006.

4. Chiu Y-C, Huang C-H, Lee T-J, Liu W-R, Yeh Y-T, Jang S-M, et al. Eu ${ }^{2+}$-activated silicon-oxynitride $\mathrm{Ca}_{3} \mathrm{Si}_{2} \mathrm{O}_{4} \mathrm{~N}_{2}$ : a green-emitting phosphor for white LEDs. Opt Exp. 2011; 19:A331-A339.

5. Jackson $\mathrm{T}$ B, Virkar A V, More $\mathrm{K}$ L, Dinwiddie $\mathrm{R}$ B, Cutler $\mathrm{R}$ A. High-Thermal-Conductivity Aluminum Nitride Ceramics: The Effect of Thermodynamic, This article is protected by copyright. All rights reserved. 
Kinetic, and Microstructural Factors. J Am Ceram Soc. 1997; 80:1421-1435.

6. Hagen E, Yu Y, Grande T, Høier R, Einarsrud M-A. Sintering of AlN Using CaO- $\mathrm{Al}_{2} \mathrm{O}_{3}$ as a Sintering Additive: Chemistry and Microstructural Development. J Am Ceram Soc. 2002; 85:2971-2976.

7. Taniyasu Y, Kasu M, Makimoto T. An aluminium nitride light-emitting diode with a wavelength of 210 nanometres. Nature 2006; 441:325-328.

8. Liu Q, Zhang L, Li J, Zhao K. Synthesis of AlN: Eu ${ }^{2+}$ green phosphors by a simple direct nitridation method. Luminescence 2017; 32:680-684.

9. Wang L, Wang X, Takeda T, Hirosaki N, Tsai Y-T, Liu R-S, et al. Structure, Luminescence, and Application of a Robust Carbidonitride Blue Phosphor $\left(\mathrm{Al}_{1-\mathrm{x}} \mathrm{Si}_{\mathrm{x}} \mathrm{C}_{\mathrm{x}} \mathrm{N}_{1-\mathrm{x}}: \mathrm{Eu}^{2+}\right)$ for Near UV-LED Driven Solid State Lighting. Chem Mater. 2015; 27:8457-8466.

10. Hu W-W, Zhu Q-Q, Hao L-Y, Xu X, Agathopoulos S. Luminescence properties and energy transfer in AlN: $\mathrm{Ce}^{3+}, \mathrm{Tb}^{3+}$ phosphors. Mater Res Bull. 2014; 51:224-227.

11. Wang X-J, Xie R-J, Dierre B, Takeda T, Suehiro T, Hirosaki N, et al. A novel and high brightness $\mathrm{AlN}: \mathrm{Mn}^{2+}$ red phosphor for field emission displays. Dalton Trans. 2014; 43:6120-6127.

12. Liu T-C, Kominami H, Greer H F, Zhou W, Nakanishi Y, Liu R-S. Blue Emission by Interstitial Site Occupation of $\mathrm{Ce}^{3+}$ in AlN. Chem Mater. 2012; 24:3486-3492.

13. Hirosaki N, Xie R J, Inoue K, Sekiguchi T, Dierre B, Tamura K. Blue-emitting AlN: Eu ${ }^{2+}$ nitride phosphor for field emission displays. Appl Phys Lett. 2007;91:3.

14. Inoue K, Hirosaki N, Xie R J, Takeda T. Highly Efficient and Thermally Stable Blue-Emitting AlN:Eu ${ }^{2+}$ Phosphor for Ultraviolet White Light-Emitting Diodes. J Phys Chem

This article is protected by copyright. All rights reserved. 
C. 2009;113:9392-9397.

15. Dierre B, Yuan X L, Inoue K, Hirosaki N, Xie R J, Sekiguchi T. Role of Si in the Luminescence of AlN:Eu,Si Phosphors. J Am Ceram Soc. 2009; 92:1272-1275.

16. Yin L-J, Xu X, Yu W, Yang J-G, Yang L-X, Yang X-F, et al. Synthesis of Eu ${ }^{2+}$-Doped AlN Phosphors by Carbothermal Reduction. J Am Ceram Soc. 2010; 93:1702-1707.

17. Yin L J, Yu W, Xu X, Hao L Y, Simeon A. The effects of fluxes on AlN: $\mathrm{Eu}^{2+}$ blue phosphors synthesized by a carbothermal reduction method. J Am Ceram Soc. 2011; 94:3842-3846.

18. Yin L-J, Zhu Q-Q, Yu W, Hao L-Y, Xu X, Hu F-C, et al. Europium location in the AlN: Eu green phosphor prepared by a gas-reduction-nitridation route. J Appl Phys. 2012; 111,053534 .

19. Shannon R D. Revised effective ionic radii and systematic studies of interatomic distances in halides and chalcogenides. Acta Crystallogr A. 1976; 32:751-767.

20. Loughin S, French R H, Ching W Y, Xu Y N, Slack G A. Electronic structure of aluminum nitride: Theory and experiment. Appl Phys Lett. 1993; 63:1182-1184.

21. Fang C M, Metselaar R, Hintzen, H T, de With, G. Structure Models for $\gamma$-Aluminum Oxynitride from Ab Initio Calculations. J Am Ceram Soc. 2001; 84: 2633-2637.

22. PENTALERI E A, GUBANOV V A, BOEKEMA C, FONG C Y. First-principles band-structure calculations of $\mathrm{p}$ - and n-type substitutional impurities in zinc-blende aluminum nitride, Wiley-VCH: Berlin, ALLEMAGNE, 1997; Vol. 203.

23. Dobrowolska A, Dierre B, Fang C M, Hintzen H T, Dorenbos P. Thermal quenching of $\mathrm{Eu}^{2+}$ emission in Ca- and $\mathrm{Sr}_{-} \mathrm{Ga}_{2} \mathrm{~S}_{4}$ in relation with VRBE schemes. J. Lumin. 2017;

This article is protected by copyright. All rights reserved. 
$184: 256-261$.

24. Fang C M, Biswas K. Quaternary Iodides $\mathrm{A}(\mathrm{BaSr}) \mathrm{I}_{5}: \mathrm{Eu}^{2+}(\mathrm{A}=\mathrm{K}, \mathrm{Cs})$ as Scintillators for Radiation Detection. J Phys Chem C. 2016;120:1225-1236.

25. Fang C, Biswas K. Preferential Eu Site Occupation and Its Consequences in the Ternary Luminescent Halides $\mathrm{AB}_{2} \mathrm{I}_{5}: \mathrm{Eu}^{2+}(\mathrm{A}=\mathrm{Li}-\mathrm{Cs} ; \mathrm{B}=\mathrm{Sr}, \mathrm{Ba})$. Phys Rev Appl. 2015; 4:014012.

26. Kresse G, Furthmüller J. Efficiency of ab-initio total energy calculations for metals and semiconductors using a plane-wave basis set. Comp Mater Sci. 1996; 6:15-50.

27. Kresse G, Hafner J. Ab initio. Phys Rev B. 1994; 49:14251-14269.

28. Blöchl P E. Projector augmented-wave method. Phys Rev B. 1994; 50: 17953-17979.

29. Kresse G, Joubert, D. From ultrasoft pseudopotentials to the projector augmented-wave method. Phys Rev B. 1999; 59:1758-1775.

30. Perdew J P, Burke K, Ernzerhof M. Generalized Gradient Approximation Made Simple. Phys Rev Lett. 1996; 77:3865-3868.

31. Monkhorst H J, Pack J D. Special points for Brillouin-zone integrations. Phys Rev B. $1976 ; 13: 5188-5192$.

32. Takeda T, Hirosaki N, Xie R-J, Kimoto K, Saito M. Anomalous Eu layer doping in Eu, Si co-doped aluminium nitride based phosphor and its direct observation. J Mater Chem. 2010; 20:9948-9953.

33. Wang Y, Xu X, Yin L, Hao L. High Thermal Stability and Photoluminescence of Si-N-Codoped BaMgAl ${ }_{10} \mathrm{O}_{17}: \mathrm{Eu}^{2+}$ Phosphors. J Am Ceram Soc. 2010; 93:1534-1536.

34. Vetter U, Hofsass H, Taniguchi T. Visible cathodoluminescence from Eu-implanted single- and polycrystal c-BN annealed under high-temperature, high-pressure conditions.

This article is protected by copyright. All rights reserved. 
Appl Phys Lett. 2004; 84:4286-4288.

35. Strite S, Morkoç H. GaN, AlN, and InN: A review. J. Vac. Sci. Technol. B: Microelectronics and Nanometer Structures Processing, Measurement, and Phenomena. 1992; 10:1237-1266.

36. Natali F, Ludbrook B, Galipaud J, Plank N, Granville S, Preston A, et al. Epitaxial growth and properties of GdN, EuN and SmN thin films. Phys status solidi (c) 2012; 9:605-608.

37. Jones R O. Density functional theory: Its origins, rise to prominence, and future. Rev Mod Phys. 2015;87:897-923.

38. Davis C S, Novikov S V, Cheng T S, Campion R P, Foxon C T. Surface reconstruction patterns of AlN grown by molecular beam epitaxy on sapphire. J Cryst Growth 2001; 226:203-208.

39. Lee C D, Dong Y, Feenstra R M, Northrup J E, Neugebauer J. Growth and Surface Reconstructions of AlN(0001) Films. Mat Res Soc Symp Proc. 2004; 798 (Y3.5.1).

40. Hikari S, Rie T, Hisashi M, Yoshinao K, Akinori K. Theoretical Analysis for Surface Reconstruction of AIN and InN in the Presence of Hydrogen. Jpn J Appl Phys. 2007; 46:5112.

41. Holec D, Mayrhofer P H. Surface energies of AlN allotropes from first principles. Scripta Mater. 2012;67:760-762.

42. Watanabe H, Yamane H, Kijima N. Crystal structure and luminescence of $\mathrm{Sr}_{0.99} \mathrm{Eu}_{0.01} \mathrm{AlSiN}_{3}$. J Solid State Chem. 2008; 181:1848-1852.

43. Li H L, Xie R J, Hirosaki N, Takeda T, Zhou G H. Synthesis and Luminescence Properties of Orange-Red-Emitting $\mathrm{M}_{2} \mathrm{Si}_{5} \mathrm{~N}_{8}: \mathrm{Eu}^{2+}(\mathrm{M}=\mathrm{Ca}, \mathrm{Sr}$, Ba) Light-Emitting Diode This article is protected by copyright. All rights reserved. 
Conversion Phosphors by a Simple Nitridation of $\mathrm{MSi}_{2}$. Int J Appl Ceram Tec. 2009; 6:459-464.

\section{Figure captions:}

Figure 1. Cathodoluminescence spectra of the AlN: Eu phosphor.

Figure 2. Eu L $\mathrm{LIII}_{-}$edge XANES spectrum of the AlN: Eu sample.

Figure 3. (a) HAADF image along [010] zones showing Eu sites along the boundary. (b) Enlarged HAADF image. (c) Intensity profile corresponding to the box in (c). (d) Simulated HAADF image showing that the intensity difference between different atoms. (e, f) another region showing Eu sites close to the boundary.

Figure 4. Relationships between the calculated energies of $\operatorname{EuX}(X=\mathrm{O}, \mathrm{N})$ crystals on the magnetic moment per Eu. The energies for moment $=7$ are used as the references. Clearly, for $X=O$, the state with $s=7 / 2$ is most stable while for $X=N, s=6 / 2$ is the ground state. That corresponds well to the ionic model: $\mathrm{Eu}^{3+} \mathrm{N}^{3-}$ and $\mathrm{Eu}^{2+} \mathrm{O}^{2-}$.

Figure 5. Total density of states (tDOS) for bulk AlN with wurtzite structure (a), for Eu doped system (b) and for Eu-O co-doped AlN system (d). The partial density of Eu 4f for Eu in AlN (c) and for $\mathrm{Eu}(\mathrm{f})$ and $\mathrm{O}$ (e) in the co-doped Eu-O (pair) system. The Fermi levels are indicated by the dotted lines respectively. The present study shows the $\mathrm{Eu}^{3+}\left(4 \mathrm{f}^{6}\right.$ configuration) in the single doping system and $\mathrm{Eu}^{2+}\left(4 \mathrm{f}^{7}\right.$ configuration) for the Eu-O coped system.

Figure 6. Schematic structure of Eu at the center (a), subsurface (b) and at surface (c) of the AlN slab, and one Eu-O pair replacing an Al-N pair near the surface (d). The small silvery spheres for Al, small yellow for $\mathrm{N}$, large oranges for $\mathrm{Eu}$, and middle blue for $\mathrm{O}$.

Figure 7. The calculated total density of states (tDOS) for Eu at AlN(110) surface (a) and the related Eu 4f states (b); total density of states (tDOS)for Eu-O at AlN(110) surface (c) and the related $\mathrm{Eu}$ 4f states (d). The dotted redline means Fermi level. The positive values means for spin-up (majority) electrons and the negative values for the spin-down (minority electrons). Clearly, the Eu at $\mathrm{AlN}$ (110) surface has $\mathrm{Eu}^{3+}$ valence and corresponding to spin states $~ 6.1 / 2$, whereas at the surface $\mathrm{Eu}^{2+}$ with spin state $\mathrm{s}=7 / 2$ for the $\mathrm{Eu}-\mathrm{O}$ pair.

This article is protected by copyright. All rights reserved. 
Figure 8. A schematic diagram showing the origin of the observed green and red emissions in AlN: Eu phosphor.

Table 1. Calculated results for binary AlN, related EuO and EuN phases using the PBE / $\mathrm{PBE}+\mathrm{U}(U=2.5 \mathrm{eV}$ for $\mathrm{Eu} 4 \mathrm{f})$ method ${ }^{23,24,25}$. The experimental values of AlN, EuN and EuO from literature are included in parentheses. SG in column $2^{\text {nd }}$ represents for space group, Mag in $5^{\text {th }}$ column for magnetic moment in the Eu sphere.

\begin{tabular}{|l|l|l|l|l|}
\hline Phases & $\begin{array}{l}\text { Lattice } \\
(\mathrm{SG})\end{array}$ & Parameter & Bonds/charges & Local moment \\
\hline Hex_AlN & $\begin{array}{l}\mathrm{P} 63 \mathrm{mc} \\
(\mathrm{nr} .194)\end{array}$ & $\begin{array}{l}a=3.128(3.112)^{35} \\
c=5.017(4.982)^{35}\end{array}$ & Al-N:1.90(×3),1.91 & 0 \\
\hline EuN* & $\begin{array}{l}\text { Fm-3m } \\
(\mathrm{nr} .225)\end{array}$ & $a=5.131(5.144)^{36}$ & Eu-N: 2.56(×6) & $\mathrm{M}_{\mathrm{mag}}=6 \mu_{\mathrm{B}} / \mathrm{Eu}$ \\
\hline EuO* & $\begin{array}{l}\text { Fm-3m } \\
(\mathrm{nr} .225)\end{array}$ & $a=5.130(5.141)^{36}$ & Eu-O: $2.55(\times 4)$ & $\mathrm{M}_{\mathrm{mag}}=7 \mu_{\mathrm{B}} / \mathrm{Eu}$ \\
\hline
\end{tabular}

Table 2. The calculated results (formation energy, $\Delta E=E\left(\mathrm{Al}_{64-\mathrm{x}} \mathrm{Eu}_{\mathrm{x}} \mathrm{N}_{64-\mathrm{y}} \mathrm{O}_{\mathrm{y}}\right)$ $-\left[E\left(\mathrm{Al}_{64-\mathrm{x}} \mathrm{Eu}_{\mathrm{x}} \mathrm{N}_{64-\mathrm{y}} \mathrm{O}_{\mathrm{y}}\right)+x E(\mathrm{EuN})+\mathrm{y} E(\mathrm{EuO})\right]$, and local chemical bonding and local spin state). Column 1 of rows 3 and 4, the symbol $\mathrm{f}$-/c- represents the co-doped $\mathrm{O}$ and Eu being far away/being close (Eu-O pair).

\begin{tabular}{|c|c|c|}
\hline Configuration & Eu bonds & $\Delta E(\mathrm{eV} / \mathrm{Eu})$ spin per $\mathrm{Eu}$ \\
\hline $\mathrm{Al}_{63} \mathrm{EuN}_{64}$ & Eu-N:2.22(×3), 2.26 & $\begin{array}{l}+2.646 \mathrm{eV} / \mathrm{EuN} \\
s=6.04 / 2\end{array}$ \\
\hline $\mathrm{f}-\mathrm{Al}_{63} \mathrm{EuN}_{63} \mathrm{O}$ & Eu-N:2.28(×3), 2.33 & $\begin{array}{l}+4.510 \mathrm{eV} / \mathrm{EuO} \\
s=7 / 2\end{array}$ \\
\hline $\mathrm{c}-\mathrm{Al}_{63} \mathrm{EuN}_{63} \mathrm{O}$ & Eu-N:2.26(×3),O:2.33 & $\begin{array}{l}+3.635 \mathrm{eV} / \mathrm{EuO} \\
s=7 / 2\end{array}$ \\
\hline
\end{tabular}

This article is protected by copyright. All rights reserved. 
Table 3. The calculated energetics for one Eu atom/ion and one Eu-O pair at different positions at/in AlN (0001) surfaces and (110).

\begin{tabular}{|c|c|c|c|}
\hline Position of Eu & $\begin{array}{l}\text { Spin state } s=(\text { Bohr } \\
\text { unit })\end{array}$ & Bond $(\AA)$ & $\Delta E(\mathrm{eV}) *$ \\
\hline \multicolumn{4}{|c|}{ AlN(110) (non-polar) } \\
\hline Eu at center & $6.26 / 2 / 7 / 2 *$ & Eu-N:2.24(×2),2.27,2.30 & $0.000 /+0.49 * *$ \\
\hline Eu at $2^{\text {nd }}$ layer & $6.29 / 2 / 7 / 2 *$ & Eu-N: $2.26(\times 2), 2.31,2.40$ & $-0.52 /-0.31 * *$ \\
\hline Eu at surface & $\begin{array}{l}5.92 / 2 / 7 / 2 * \\
7 / 2\end{array}$ & $\begin{array}{l}\text { Eu-N: } 2.33,2.366(\times 2) \\
\text { Eu-N:2.26,2.29 } \\
\text {-O: } 2.59\end{array}$ & $\begin{array}{l}-3.05 /-3.02 * * \\
-3.22 /(\mathrm{EuO}) * *\end{array}$ \\
\hline $\mathrm{EuO}$ at center & $7 / 2$ & $\begin{array}{l}\text { Eu-N:2.27,2.29,2.31 } \\
\text {-O: } 2.31\end{array}$ & 0.0 \\
\hline \multicolumn{4}{|c|}{ AlN(0001) (polar) } \\
\hline Eu at center & $/ 7 / 2 *$ & Eu-N:2.16(×3), 2.37 & 0.0 \\
\hline $\begin{array}{l}\text { Eu at Al_surf } \\
\text { Eu at N_surf }\end{array}$ & $\begin{array}{l}/ 7 / 2 * \\
/ 7 / 2 *\end{array}$ & $\begin{array}{l}\text { Eu-N: } 2.41(\times 3) \\
\text { Eu-N: } 2.16(\times 3)\end{array}$ & $\begin{array}{l}-4.72 * * \\
-1.91 * *\end{array}$ \\
\hline
\end{tabular}

$*$ indicates the spin is fixed to $s=7 / 2$ and $* *$ is the related energy differences with respect to the ones of zero eV.

This article is protected by copyright. All rights reserved. 

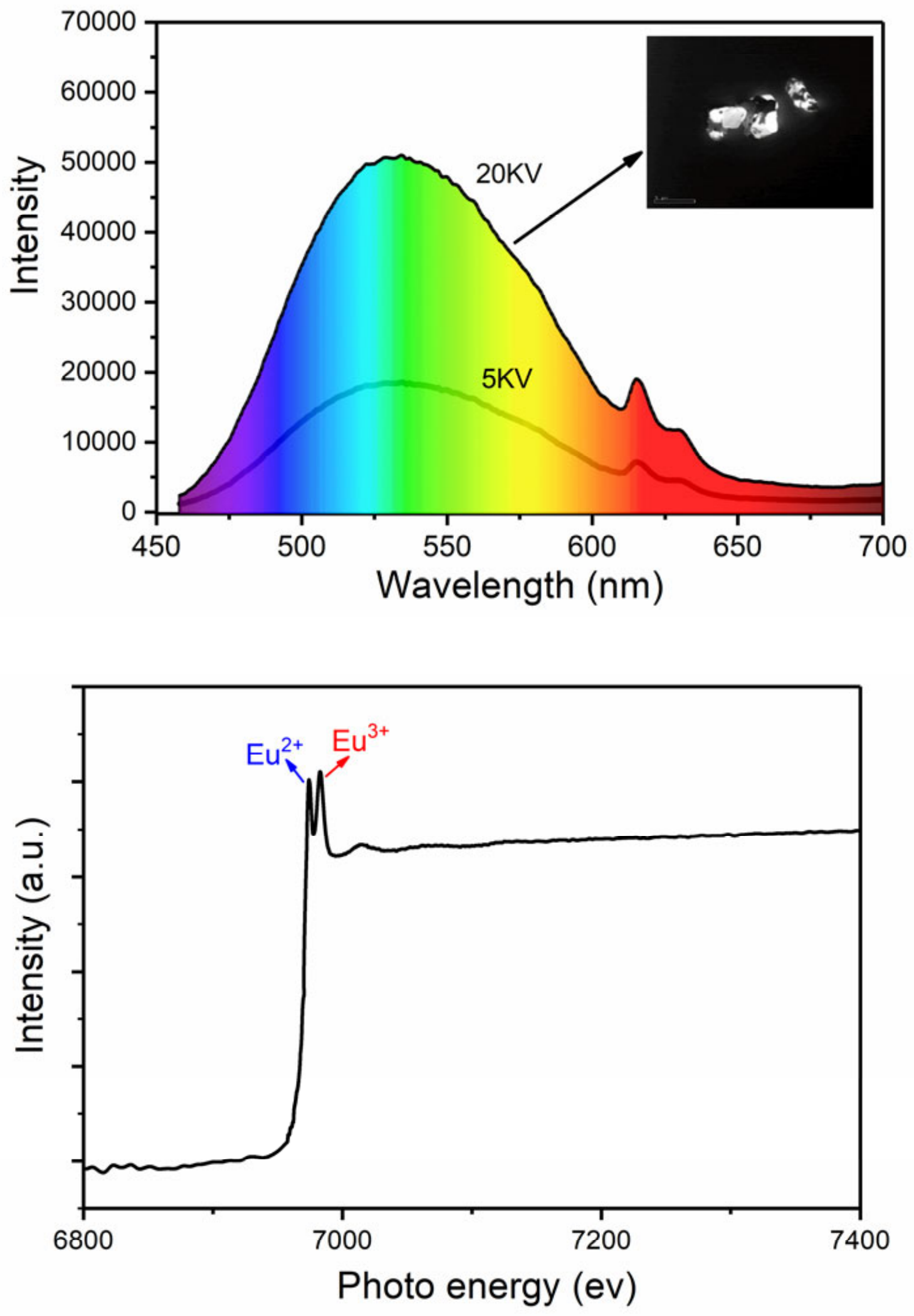

This article is protected by copyright. All rights reserved. 

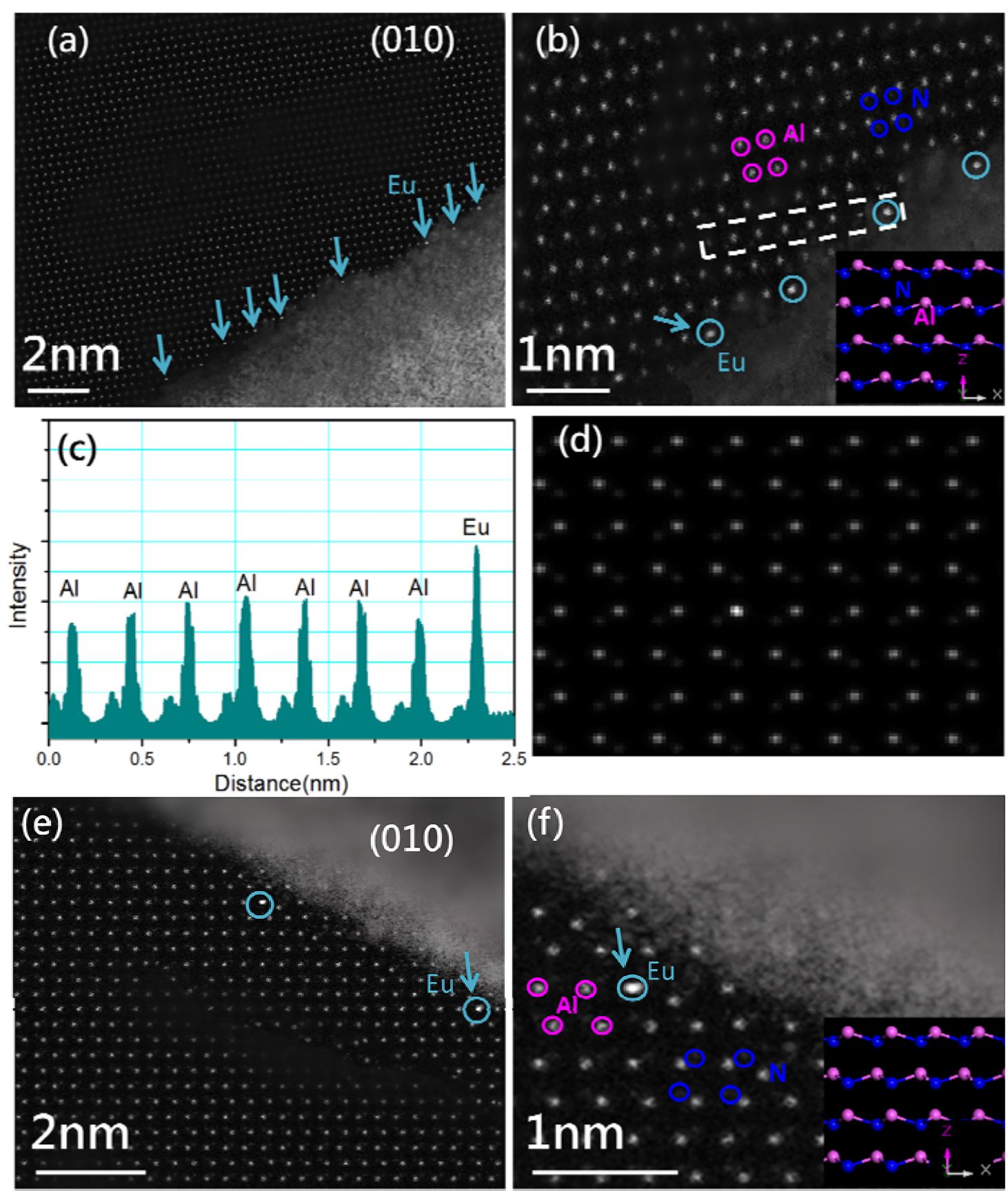

This article is protected by copyright. All rights reserved. 

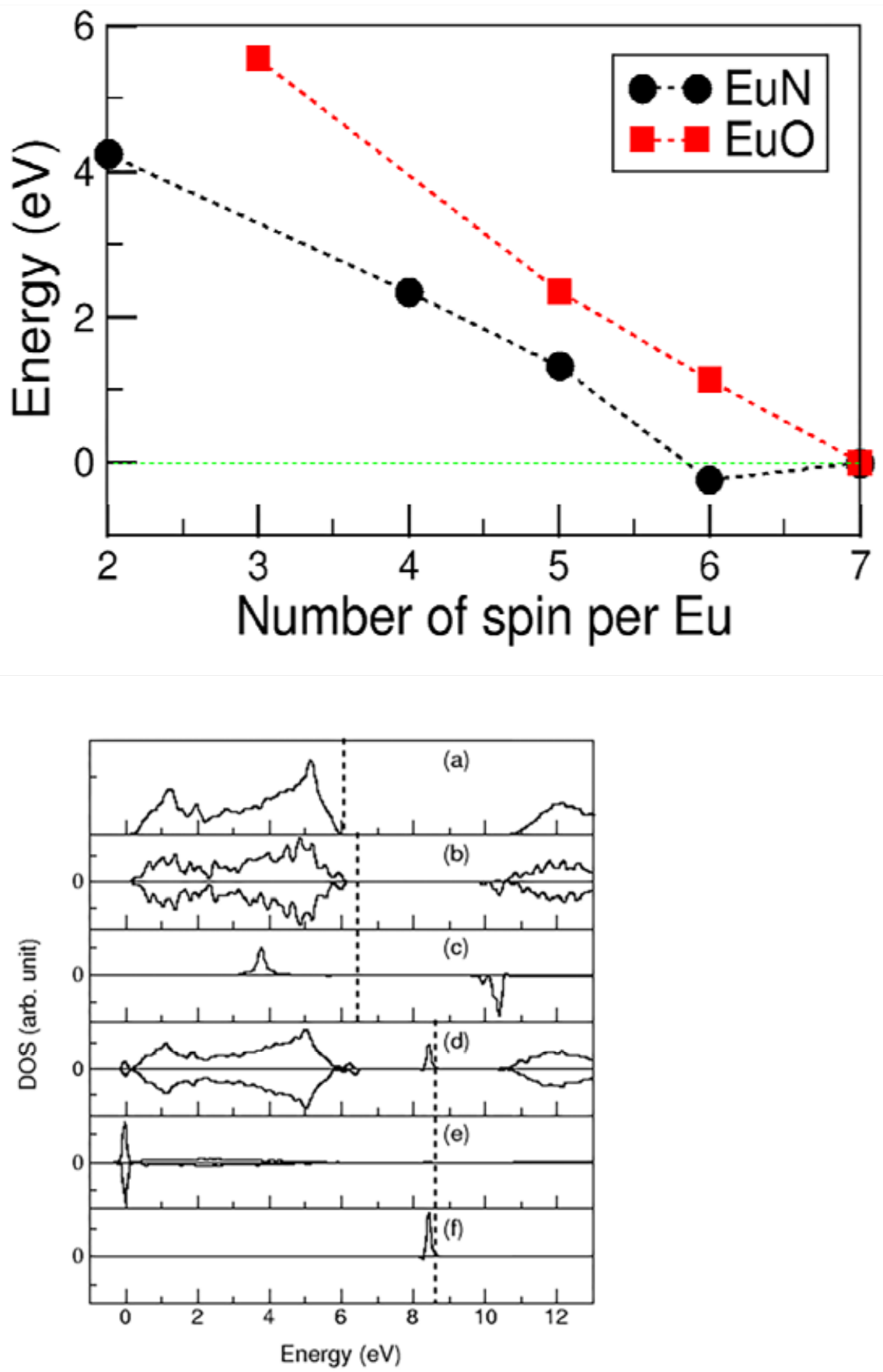

This article is protected by copyright. All rights reserved. 

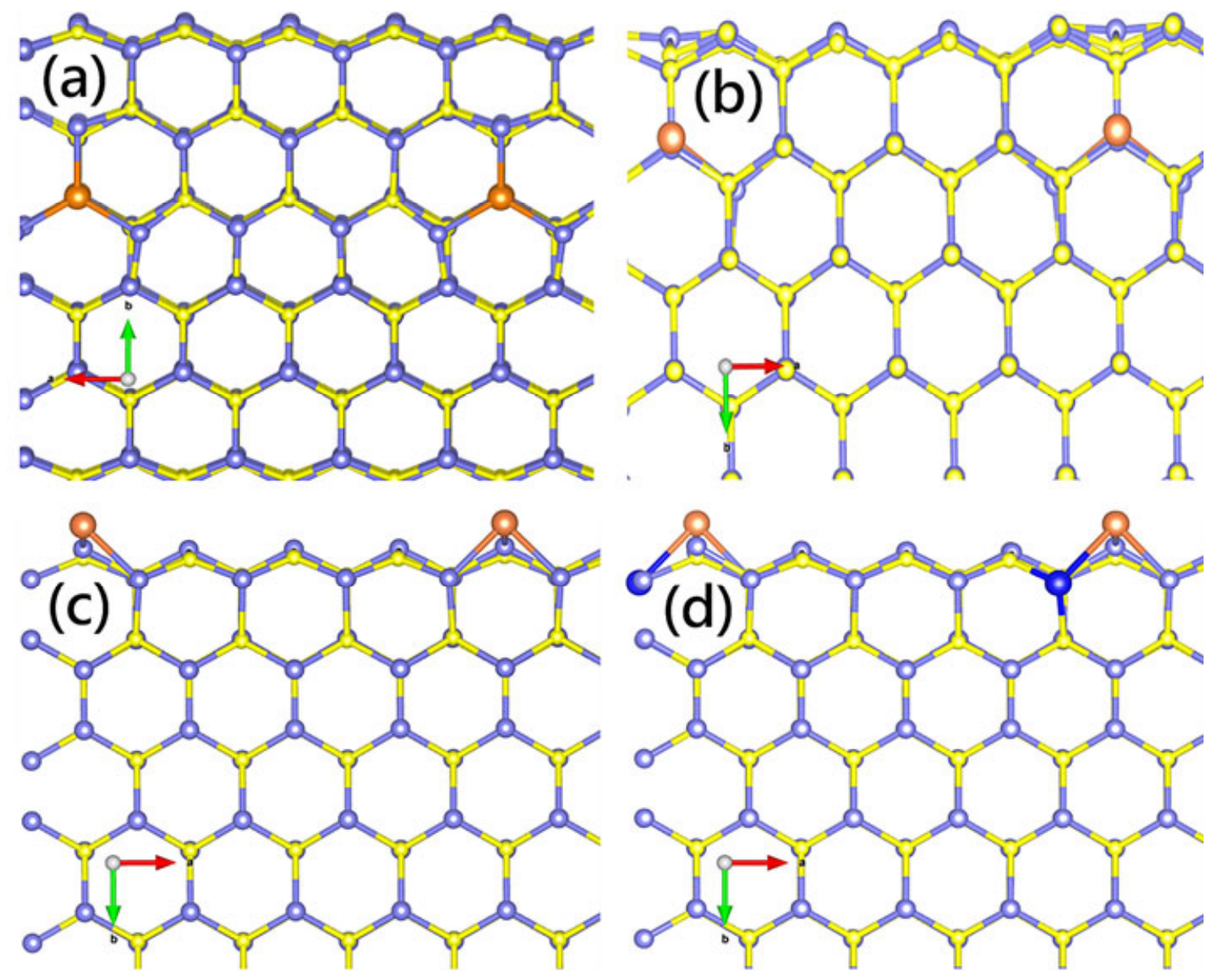

This article is protected by copyright. All rights reserved. 

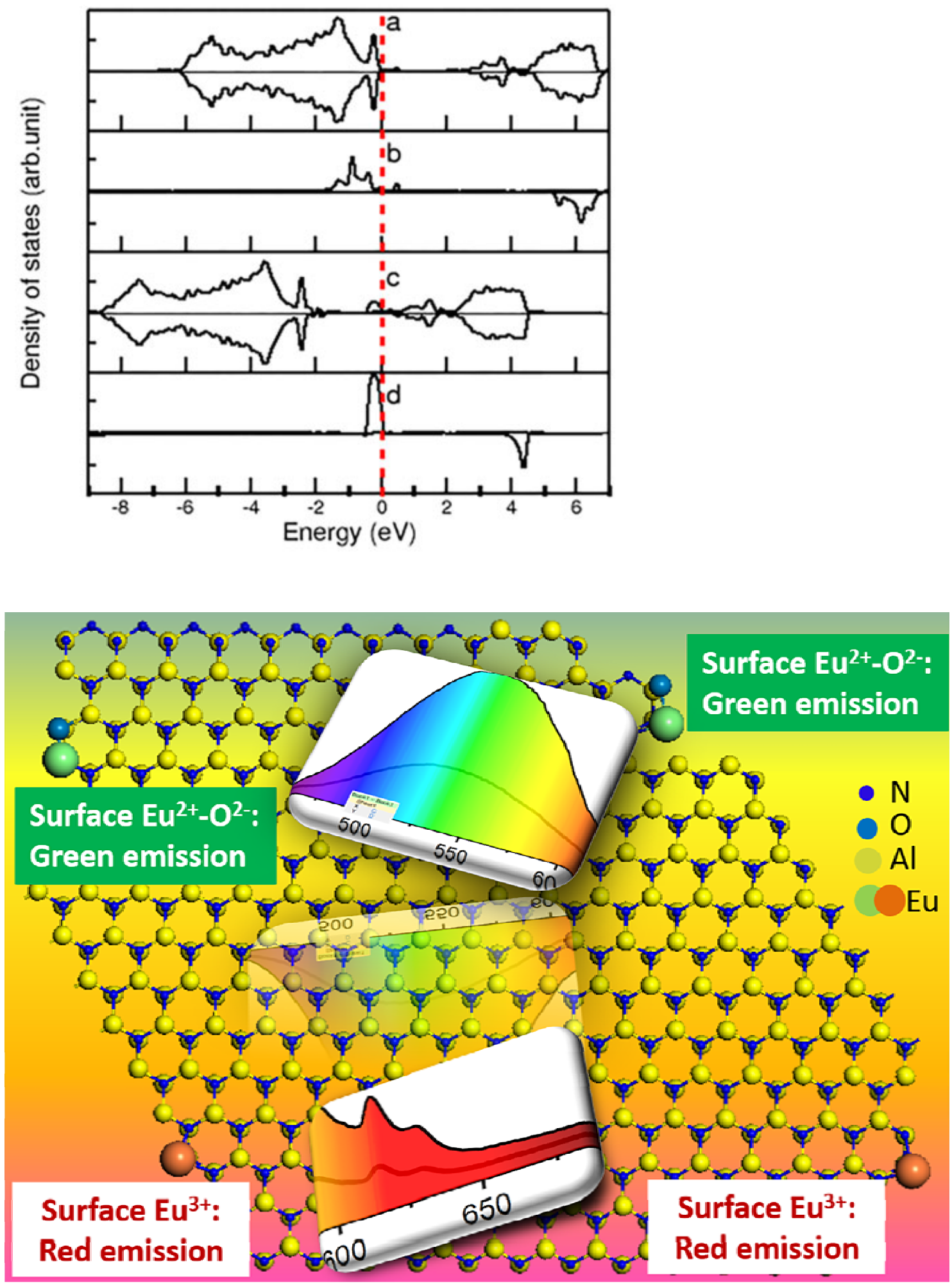

This article is protected by copyright. All rights reserved. 